\section{Die nCPAP-Therapie beeinflusst nicht die Atemmechanik von Patienten mit schlafbezogenen Atmungsstörungen}

\author{
Lungenfunktion unter nCPAP
}

\section{H. Hein, H. Magnussen}

Zentrum für Pneumologie und Thoraxchirurgie, Krankenhaus Großhansdorf
Zusammenfassung: Wir untersuchten bei Patienten mit schlafbezogenen Atmungsstörungen den langfristigen Effekt einer nCPAP-Therapie auf die Atemmechanik und den Gasaustausch. 109 Patienten (19 Frauen, 90 Männer; Alter [Mittelwert/ Standardabweichung] 59 \pm 10 Jahre, Apnoe-Hypopnoe-Index vor Therapie $34 \pm 24 /$ Std.) wurden für $27 \pm 23$ Monate mit nCPAP (7,7 \pm 2 mbar) behandelt. Die Nachuntersuchungen erfolgten nach mindestens 6 Monaten. Die Therapie wurde durchschnittlich 5,6 $\pm 1,9$ Std./Nacht genutzt. Vitalkapazität, $\mathrm{FEV}_{1}$, intrathorakales Gasvolumen und Blutgasanalysen wurden unter Spontanatmung vor und unter Therapie gemessen. Statistisch signifikante Änderungen (Wilcoxon-Test) waren weder für die Vitalkapazität, die $\mathrm{FEV}_{1}$, das thorakale Gasvolumen, $\mathrm{PaO}_{2}$ oder $\mathrm{PaCO}_{2}$ nachweisbar. Lediglich 6 vor Therapie hypoxämische Patienten zeigten einen Anstieg des $\mathrm{PaO}_{2}$ von $63 \pm 4$ auf $70 \pm 6 \mathrm{~mm} \mathrm{Hg}(\mathrm{p}<0,01)$. Die getrennte Analyse der Raucher, Exraucher und Nichtraucher zeigte keine statistisch signifikanten Änderungen der Lungenfunktionsparameter. Die Berücksichtigung der Höhe des CPAP-Wertes und der Nutzungsdauer hatte keinen Einfluss auf die Ergebnisse. Wir schließen aus unseren Daten, dass der längerfristige Einsatz von nCPAP zu keinen signifikanten Änderungen der Lungenfunktion und der kapillären Blutgase führt. Eine Hypoxämie kann sich bessern.

Schlüsselwörter: Schlafbezogene obstruktive Atmungsstörungen - nCPAP-Therapie - Lungenfunktion

The nCPAP Therapy does not Influence Respiratory Mechanism of Patients with Sleep-Related Respiratory Disturbances: We investigated in patients with sleep disordered breathing the influence of long-term nCPAP-therapy on airway mechanics and capillary blood gases. 109 patients (19 women, 90 men, age [mean \pm standard deviation] $59 \pm 10$ years, apneahypopnea-index $34 \pm 24 / \mathrm{h}$ ) were treated for $27 \pm 33$ months, with a mean pressure of $7.7 \pm 2$ mbar, mean usage $5.6 \pm 1.9 \mathrm{~h} /$ night. Vital capacity, $\mathrm{FEV}_{1}$, thoracic gas volume and capillary blood gases did not change during therapy, irrespective of the smoking history. However, in a subgroup of 6 patients who were hypoxemic before therapy, there was a significant increase of $\mathrm{PaO}_{2}$ from $63 \pm 4$ to $70 \pm 6 \mathrm{~mm} \mathrm{Hg}(p<0.01)$. Neither the height of the pressure nor the duration of therapy did influence the course of lung function data.

Pneumologie 2000; 54: 335-337

(c) Georg Thieme Verlag Stuttgart · New York ISSN 0934-8387
Key words: Sleep disordered breathing - Lung function nCPAP-therapy

\section{Einleitung}

Patienten mit schlafbezogenen Atmungsstörungen haben in der Regel Lungenfunktionswerte, die sich von Kontrollpersonen nicht unterscheiden [1]. Bisherige Untersuchungen zeigten unter einer nCPAP-Therapie [2] weder kurz [3] noch langfristige $[4,5,6]$ signifikante Änderungen, allerdings wurde in einer Untersuchung eine geringe Reduktion der $\mathrm{FEV}_{1}$ [7] beobachtet. Neben einem eventuellen Einfluss der Beatmungstherapie hat auch das Rauchverhalten Bedeutung. Wir überprüften den zeitlichen Verlauf der Lungenfunktionswerte bei Patienten unter nCPAP-Therapie in Abhängigkeit von der Dauer der Behandlung und dem Raucherstatus.

\section{Methodik}

Wir untersuchten den Verlauf der spirometrischen und bodyplethysmographischen Lungenfunktionswerte sowie von kapillären Blutgasanalysen bei 109 Patienten. Von den untersuchten 19 Frauen und 90 Männern waren 57 Nichtraucher, 29 Exraucher und 23 Raucher. Das Alter lag bei Mittelwert \pm Standardabweichung) $59 \pm 10$ Jahren, der Apnoe-HypopnoeIndex betrug vor Therapie $34 \pm 24 /$ Std., der nCPAP-Wert 7,7 \pm 2 mbar (Tab. 1).

Tab. 1 Geschlechtsbezogene Anzahl der Untersuchten, Alter, Gewicht, Bodymass-Index (BMI), Zigarettenkonsum (pack-years), CPAPWert, durchschnittliche tägliche Nutzungsdauer der CPAP-Therapie und Dauer der CPAP-Therapie aller Untersuchten unterteilt in Ex-/Nichtraucher sowie Raucher. (p: Signifikanzniveau; n. s.: nicht signifikante Unterschiede)

\begin{tabular}{llll}
\hline & Ex-/Nichtraucher & Raucher & $\mathrm{P}$ \\
\hline Frauen & 16 & 3 & \\
Männer & 70 & 20 & \\
Alter (Jahre) & $60 \pm 10$ & $54 \pm 10$ & n.s. \\
Gewicht $(\mathrm{kg})$ & $93 \pm 15$ & $91 \pm 17$ & n.s. \\
Bodymass-Index $\left(\mathrm{kg} / \mathrm{m}^{2}\right)$ & $30 \pm 5$ & $30 \pm 5$ & n.s. \\
pack-years & $9 \pm 15$ & $32 \pm 20$ & 0,0001 \\
CPAP (mbar) & $7,7 \pm 2$ & $7,7 \pm 1,6$ & n.s. \\
Nutzung/Nacht (Std.) & $5,6 \pm 1,9$ & $5,1 \pm 1,8$ & n.s. \\
Dauer (Monate) & $29 \pm 25$ & $17 \pm 12$ & 0,031 \\
\hline
\end{tabular}


Anlässlich routinemäßig durchgeführter polysomnographischer Nachuntersuchungen füllten die Patienten einen standardisierten Nebenwirkungsfragebogen aus und der Stand des Betriebsstundenzählers des CPAP-Gerätes wurde notiert.

Die Lungenfunktionsdaten wurden am Master-Lab (Fa. E. Jäger, Höchberg) spirometrisch (Vital- und $\mathrm{FEV}_{1}$, unter ATSBedingungen [8]) und mittels Bodyplethysmograph (intrathorakales Gasvolumen) gemessen. Blutgasanalysen erfolgten aus kapillären Blutproben, die dem mit Finalgon ${ }^{\circledR}$ hyperämisierten Ohrläppchen entnommen wurden und im CibaCorning-Analysator Nr.278 (Ciba-Corning, Medfield, MA, USA) gemessen wurden.

Geprüft wurden Unterschiede zwischen beiden Untersuchungszeitpunkten mittels des Wilcoxon-Tests, ein $\mathrm{p}<0,05$ galt als signifikant.

\section{Ergebnisse}

Die nCPAP-Therapie wurde über $27 \pm 23$ Monate durchschnittlich 5,6 \pm 1,9 Std./Nacht durchgeführt (Tab. 1).

Statistisch signifikante Änderungen waren weder für die Vitalkapazität, die $\mathrm{FEV}_{1}$, das intrathorakale Gasvolumen, den Atemwegswiderstand, den $\mathrm{PaCO}_{2}$ noch den $\mathrm{PaO}_{2}$ nachweisbar (Tab. 2). Auch bei getrennter Betrachtung der Patienten mit und ohne Atemwegsobstruktion vor nCPAP-Therapie (Grenzwert: $\mathrm{FEV}_{1}<70 \%$ des Solls) sind die gemessenen Änderungen der $\mathrm{FEV}_{1}$ in beiden Kollektiven ähnlich $\left(\mathrm{FEV}_{1}<70 \%\right.$ des Solls, $\mathrm{n}=10$, Zunahme um 0,06l; $\mathrm{FEV}_{1}>70 \%$ des Solls, $\mathrm{n}=99$, Abnahme um $0,1 \mathrm{l}$; n.s.). Lediglich 6 vor Therapie hypoxämische Patienten (2 Raucher, 1 Exraucher, 3 Nichtraucher) zeigten einen Anstieg des Sauerstoffpartialdruckes, von $63 \pm 4$ auf $70 \pm 6 \mathrm{~mm} \mathrm{Hg}(\mathrm{p}<0,01)$. Auch eine Subgruppenanalyse der Raucher, Exraucher und Nichtraucher zeigte keine statistisch signifikante Änderung der Parameter. Auch unter Berücksichtigung der Höhe des CPAP-Wertes und der Benutzungsdauer waren keine statistisch signifikanten Änderungen erkennbar.

\section{Diskussion}

Wir schließen uns unseren Daten, dass keine signifikanten Langzeitveränderungen der Lungenfunktion unter einer nCPAP-Therapie auftreten.

Auch andere Untersucher beschrieben keine signifikanten Änderungen der Lungenfunktionswerte während einer
nCPAP-Therapie [4,5,6]. Lediglich Chaouat et al. [7] fanden nach 5-jähriger CPAP-Therapie einen geringen aber signifikanten Rückgang der $\mathrm{FEV}_{1}$ von $80 \pm 21$ auf $76 \pm 21 \%$ des Solls. Diese Daten basieren aber im Unterschied zu unseren Ergebnissen auf einer längeren Beobachtungsdauer (5 Jahre vs. 27 Monate), der Anteil an Rauchern war deutlich höher (77\% vs. $27 \%$ ) und der beobachtete $\mathrm{FEV}_{1}$-Abfall entspricht dem durchschnittlichen Abfall von Patienten mit COPD [9].

Auch unter Berücksichtigung des Rauchverhaltens sind in unseren Subkollektiven keine signifikanten Änderungen erkennbar. Somit führt die nCPAP-Therapie auch nicht bei Patienten mit dem Risikofaktor Rauchen zu Störungen der Lungenfunktion. Unter nCPAP-Behandlung kann eine bronchiale Hyperreagibilität auftreten, bevorzugt bei subjektiv berichteten Reizungen der naso- und oropharyngealen Schleimhäute [10,11]. Der zugrundeliegende Mechanismus ist nicht bekannt. Da unsere Untersuchung keine Empfindlichkeitstestung der Atemwege einschloss, kann eine reversible Obstruktion in unserem Kollektiv nicht ausgeschlossen werden. Aber auch bei Unterteilung unseres Kollektives in Patienten mit und ohne Atemwegsobstruktion (FEV $<70 \%$ bzw. $>70 \%$ des Solls) vor nCPAP-Therapie sind die beobachteten Änderungen der $\mathrm{FEV}_{1}$ ungerichtet. Über Reizungen der naso- und oropharyngealen Schleimhäute berichteten $48 \%$ unserer Untersuchten im standardisierten Nebenwirkungsfragebogen, der routinemäßig anlässlich der Nachuntersuchungen vorgelegt wird.

Unter einer nCPAP-Therapie wurde von Leech et al. [4] und Sforza et al. [5] eine Zunahme der tagsüber gemessenen Sauerstoffpartialdrucke um im Mittel 13 [4] bzw. 3 mm Hg [5] beschrieben. Wie von Sforza et al. [5] dargestellt, zeigten auch in unserem Kollektiv nur die vor Therapie hypoxämischen Patienten einen signifikanten Anstieg des Sauerstoffpartialdruckes, von $63 \pm 4$ auf $70 \pm 6 \mathrm{~mm} \mathrm{Hg}(\mathrm{p}<0,01)$. Der Sauerstoffpartialdruck der übrigen Patienten blieb statistisch gleich. Leech et al. [4] beschrieben eine kontinuierliche Besserung des Sauerstoffpartialdruckes in Abhängigkeit von der Zeitdauer der CPAP-Therapie mit einer Korrelation von $r=0,58$. Wir sahen keinen derartigen Effekt, die Korrelation zwischen der Änderung des Sauerstoffpartialdruckes und der Dauer der Therapie betrug $r=-0,23$. Der einzige Patient mit Hyperkapnie hatte bis zur Nachuntersuchung $40 \mathrm{~kg}$ abgenommen, so dass die Normalisierung des $\mathrm{PaCO}_{2}$ dieses Patienten auch durch die Gewichtreduktion zu erklären ist. Schlussfolgerungen über Änderungen des $\mathrm{PaCO}_{2}$ unter CPAPTherapie können wir daher nicht ziehen.

\begin{tabular}{|c|c|c|c|c|c|c|}
\hline & $\begin{array}{l}\text { Ex-/Nichtrau } \\
\text { vor nCPAP }\end{array}$ & $\begin{array}{l}\text { her } \\
\text { unter nCPAP }\end{array}$ & $\mathrm{p}$ & $\begin{array}{l}\text { Raucher } \\
\text { vor nCPAP }\end{array}$ & unter nCPAP & $\mathrm{P}$ \\
\hline Vitalkapazität [l] & $4,2 \pm 1$ & $4,1 \pm 0,9$ & n.s. & $4,2 \pm 1$ & $4,1 \pm 1$ & n.s. \\
\hline $\mathrm{FEV}_{1}[\mathrm{I}]$ & $3,2 \pm 0,9$ & $3,1 \pm 0,8$ & n.s. & $3,1 \pm 0,9$ & $3 \pm 0,9$ & n.s. \\
\hline $\mathrm{FEV}_{1}$ (\% vom Soll) [\%] & $96 \pm 23$ & $95 \pm 23$ & n.s. & $97 \pm 23$ & $94 \pm 18$ & n.s. \\
\hline $\operatorname{Raw}\left[\mathrm{cmH}_{2} \mathrm{O}^{*} \mathrm{~s} / \mathrm{l}\right]$ & $2,6 \pm 1,1$ & $3 \pm 1,7$ & n.s. & $2,6 \pm 1,2$ & $3,2 \pm 1,5$ & n.s. \\
\hline ITGV (\% vom Soll) [I] & $107 \pm 18$ & $109 \pm 17$ & n.s. & $108 \pm 22$ & $101 \pm 23$ & n.s. \\
\hline $\mathrm{PaO}_{2}[\mathrm{~mm} \mathrm{Hg}]$ & $72 \pm 10$ & $75 \pm 7$ & n.s. & $76 \pm 9$ & $76 \pm 8$ & n.s. \\
\hline $\mathrm{PaCO}_{2}[\mathrm{~mm} \mathrm{Hg}]$ & $39 \pm 4$ & $38 \pm 4$ & n.s. & $38 \pm 5$ & $39 \pm 3$ & n.s. \\
\hline
\end{tabular}

Tab. 2 Änderung der Vitalkapazität, der $\mathrm{FEV}_{1}$, des \%-Solls FEV $\mathrm{F}_{1}$, des Atemwegswiderstandes, des \%-Solls des intrathorakalen Gasvolumens und des Sauerstoff- und Kohlendioxydpartialdruckes vor und unter CPAP-Therapie, unterteilt für Ex-/Nichtraucher sowie Raucher. (p: Signifikanzniveau; n. s. nicht signifikante Unterschiede) 
Zusammenfassend zeigen unsere Ergebnisse, dass unter einer nCPAP-Therapie bei obstruktivem Schlafapnoesyndrom die Lungenfunktionswerte unverändert bleiben, eine vorher bestehende Hypoxämie sich aber bessern kann.

\section{Danksagung}

Wir danken der Fa. G. Weinmann, Hamburg, für die Unterstützung bei dieser Studie.

\section{Literatur}

${ }^{1}$ Duchna HW, Rasche K, Orth M, Bauer TT, Schultze-Werninghaus G. Anamnestische und polygraphische Parameter bei OSASPatienten mit eingeschränkter Nasenatmung am Tage im Vergleich zu OSAS-Patienten mit normaler Nasenatmung. Wien Med Wochenschr 1996; 146: 348 - 349

${ }^{2}$ Sullivan CE, Berthon-Jones M, Issa FG, Eves L. Reversal of obstructive sleep apnoea by continous positive airway pressure applied through the nares. Lancet 1981; 1: $862-865$

${ }^{3}$ Kita H, Ohi M, Chin K, Noguchi T, Otsuka N, Tsuboi T, Kuno K. Effects of nasal continuous positive airway pressure therapy on respiratory parameters of upper airway patency in patients with obstructive sleep apnea syndrome. Chest 1998; 114: 691 -696

${ }^{4}$ Leech JA, Önal E, Lopata M. Nasal CPAP continues to improve sleep-disordered breathing and daytime oxygen over long-term follow-up of occlusive sleep apnea syndrome. Chest 1992; 102: $1651-1655$

${ }^{5}$ Sforza E, Krieger J, Weitzenblum E, Apprill M, Lampert E, Ratamaharo J. Long-term effects of treatment with nasal continous positive airway pressure on daytime lung function and pulmonary hemodynamics in patients with obstructive sleep apnea. Am Rev Respir Dis 1990; 141: 866-870

${ }^{6}$ Young IH, Mihalyka M, Cosats L, Sullivan CE. Long term lung function changes in patients with obstructive sleep apnoea during treatment with nasal continuous positive airway pressure. Thorax 1987; 42: 722

${ }^{7}$ Chaouat A, Weitzenblum E, Kessler P, Oswald M, Sforza E, Liegeon M-N, Krieger J. Five-year effects of nasal continous positive airway pressure in obstructive sleep apnoea syndrome. Eur Respir J 1997; 10: 2578 - 2582

${ }^{8}$ American Thoracic Society. Standardization of spirometry: 1994 update. Am J Respir Crit Care Med 1995; 152: 1107-1136

${ }^{9}$ Anthonisen N, Connett J, Kiley J, Altose M, Bailey W, Buist A, Conway W, Enright P, Kanner R, O Hara P. Effects of smoking intervention and the use of an inhaled anticholinergic bronchodilator an the rate of decline of $\mathrm{FEV}_{1}$. The Lung Health Study. J Am Med Assoc 1994; 272: 1497-1505

${ }^{10}$ Thalhofer S, Dorow P, Meissner P, Luding K. Änderung der bronchialen Hyperreaktivität unter nCPAP-Beatmung bei Patienten mit schlafbezogenen Atmungsstörungen. Pneumologie 1997; 51: $767-769$

${ }^{11}$ Wenzel G, Schönhofer B, Wenzel M, Köhler D. Bronchiale Hyperreagibilität und nCPAP-Therapie. Pneumologie 1997; 51: $770-$ 772
Dr. med. H. Hein

Krankenhaus Großhansdorf

Zentrum für Pneumologie und Thoraxchirurgie

Wöhrendamm 80

22927 Großhansdorf

E-mail: Holger.Hein@t-online.de 